\title{
A social actors network approach for the design of networked and virtual enterprises
}

\author{
António Lucas Soares ${ }^{1,2}$, César Augusto Toscano ${ }^{1}$, Jorge Pinho de Sousa ${ }^{1,2}$ \\ ${ }^{1}$ INESC Porto and ${ }^{2}$ Faculty of Engineering of University of Porto \\ Rua José Falcão, 110,4050-315 Porto,Portugal.e-mail: \{asoares@inescporto.pt, \\ ctoscano@inescporto.pt,jsousa@inescporto.pt\}
}

\begin{abstract}
This paper describes an approach to the technical and organisational design of networked (and virtual) enterprises using a social actors network perspective. The goal is to outline a set of methods, tools and models that support a more effective analysis, design, implementation and (re)configuration of of the organisational structure and information systems supportting networks of enterprises. This paper introduces the social actors network concept, as well as its principles and scope. The application of social actors network in the domain of networked/virtual enterprises is explained. The paper concludes with an example derived from the EU supported Co-Operate IST project.
\end{abstract}

Key words: networked and virtual enterprises, social actor networks, agent systems

\section{INTRODUCTION}

Over the past decade much attention has been paid to the relations between enterprises in order to cope with an increasingly global environment. "Supply Chain Management", "Networks of Enterprises", "Virtual Enterprise", are terms that entered into the vocabulary of every manager, engineer and scientist interested in the production of goods or services. These terms also stand for a broad and active area of research. On a technical side, the research work has been split over topics such as distributed information technology infra-structures and distributed information systems architectures, novel forms of organisational structures facilitated by those technological advances, new business processes based on inter-organisational relations, etc.

On the social and organisational side, much less research work has been done. Particularly, when it comes to consider inter-organisational co- 
operation, the focus is at the business process level, with the actors (individuals and groups) being diluted in very aggregate roles. Nevertheless, relationships between enterprises will continue to involve people in key managing activities, taking operational, tactical and strategic decisions supported by conveniently distributed information systems. In this context, we support the argument that there is a need of methods that consider the design of inter-organisational co-operation including explicitly the socialactors involved in such co-operation processes. Therefore, we propose an approach to the analysis and design of networked/virtual enterprises (N/VE) centred around the concept of social actor's networks (SAN) [3]. The main goal is to provide a conceptual and methodological tool that is able to:

1. Support the modelling of N/VE focusing on the structural aspects i.e., to know which organisational elements (social actors) are involved, how these elements are related to each other, what kind of relationships exist between elements;

2. Support the modelling of N/VE focusing on the relational aspects i.e., to characterise social actors, to characterise the relationships between social actors;

This approach is intended to be a companion to a conventional business process analysis (including modelling). While a business process modelling method (for example UML with Erikson-Penker extensions) provide a tool to analyse the distributed business processes structure in a N/VE, it does not so for issues that are most important in implementing and operating these processes: social actors and their relationships. SAN is intended to be used as a conceptual tool to question and plan a desired network of social actors concerning the structural, relational and individual aspects.

We argument that the SAN approach can be an effective approach to the analysis and design of $\mathrm{N} / \mathrm{VE}$, unifying the organisational and information technology aspects.

\section{PRINCIPLES AND SCOPE OF SAN}

SAN belong to a category of sociological methods (structural methods) where the goal is to highlight the structural relationships between social actors, enabling the contextualization of their actions in a systematic way. It is a simple representation of a system of complex relationships and exchanges between social actors [2].

More specifically, SAN are networks that connect individual and/or collective social actors. This concept is more specific than "social network" that names a very active social sciences area of research (a social network is not necessarily a SAN: take the example of a public transportation network). 
Social actors can be in contact through physical infrastructures (phone networks, data networks) and this contact is mediated by several kinds of artefacts, from phone sets to computer programs. Here our main interest is obviously computer mediated communication, but SAN can encompass, in principle, every possible form of social contact.

Within the N/VE domain, there are several types of structures (networks) that are interesting to model, going from more formal types such as client/supplier or co-operative planning, to less formal ones such as technical co-operation, informal information exchange, interest groups, etc.

\subsection{Definitions}

A set of social actors constitute a network if there is some sort of relationship between them. Social actors are represented as the nodes of the network, while the relationships are represented as arcs. Social actors are individuals or groups capable of performing activities and of interacting with other actors in order to pursue goals. Below, we will consider software agents as a class of actors eligible to be included in a SAN.

It can be useful to distinguish three general classes of relationships in a SAN : transactions, controls and links. Transactions include every kind of exchange between two social actors within a network. This can go from well defined transactions such as exchange of data to ill defined transactions such as the exchange of influence. Transactions between two actors can be symmetric if both actors can establish similar types of transactions or asymmetric if it is not the case. Control can be defined as the capacity of a social actor to decide on performing or not a given transaction with other social actor. Control can be unilateral or shared (this can be further characterised by a level of control over a given transaction). Links are the more general class and include every possible kind of relationships not falling into the previous categories. Examples of links can be attitudes such as "awareness", "hostility", "trust", being possible to distinguish between positive, negative or neutral links (or using another terminology identification, differentiation or indifference links).

\subsection{Basic levels of analysis}

The analysis of a SAN can be done at several levels. The choice of the adequate level depends obviously upon the purpose of the analysis. One possible division (inspired in [3]) is to consider the individual, relational and structural levels. This provides several abstraction or detail levels.

The structural level tries to describe and analyse globally a given network, requiring complete data about the different actors and types of 
relations. For example, for a complete supply chain to be studied at the structural level all the participating companies must be known, as well as the whole of their client-supplier relations. Such analysis would provide for example an identification of companies with the same supplying characteristics (types of products, delivery performance, supply chain tier, etc.).

The relational level concerns the characterisation of the relationships between the social actors. Some types of measures taken from graph theory (e.g., distance, accessibility) can be used to characterise these relationships. Analysis at this level can be either qualitative and quantitative. Examples of this are the evaluation of logistic issues between two supply chain commercial partners (such as delivery lead times, quality performance), the degree of trust between partners, the degree information exchange concerning several subjects (such as production plans, informal information concerning production status).

Finally, at the individual level the social actors are analysed and characterised based on the relationships in which they participate. This should be complemented with other types of characterisation of the social actors, resulting from the application of other analysis methods. Examples of analysis results are the evaluation of the importance of an actor (e.g., measured by the centrality of his position concerning information exchange in the network), the potential for an actor to establish relationships in the network, etc.

\subsection{Basic types of analysis}

At the basic levels described above, SAN approach can be used to analyse different aspects of a network of social actors. In social sciences research, SAN approach has been used for several types of analysis: communication networks, support networks, enterprise networks, policy networks, commerce networks, etc. (see e.g., [3]).

The basic types of analysis of N/VE reflect the perspective under which the analysis is done. The possible analysis types fall in two categories (1) organisational and/or technical structure analysis and (2) social interaction analysis. These two categories promote a socio-technical approach, as they can be identified with a technical and social sub-systems analysis.

\section{ANALYSIS OF SAN IN THE N/VE CONTEXT}

A network of enterprises can be made up of several different entities and types of relationships according to their relative position within e.g., the 
supply chain, and consequently may have several interaction forms working simultaneously. Interaction among participants in the network, their coordination and their access to information are becoming increasingly important. Setting up a network and managing it in an optimised way, whilst balancing customer needs with increased performance along the whole chain, may be a key factor for the competitiveness of a specific company and in overall the entire network. For a clear understanding of what are the enabling and restraining factors of effectiveness in an established network or one setting up, SAN modelling will help to analyse the complementary perspectives.

This approach is intended to be sufficiently powerful to be used in both organisational and technical design. In fact, the broad goal of our research work is to investigate tools to support the joint and optimised development of information systems and organisational processes in N/VE. Organisational processes encompass business processes as well as the individual and social processes.

We will describe now how the SAN approach can be used in the main phases of virtual/network enterprises life-cycle: creation and operation.

\subsection{Creation}

According to [1], the major steps in creating a VE or network of enterprises are: (1) setup infra-structure resources, (2) manifestation, (3) creation/evolution, (4) contract negotiation/re-negotiation, and (5) enterprise configuration/re-configuration. Phases 1 and 4 will not be considered as the SAN approach would have little to do about them. Phase 1 concerns to the definition and installation of generic computational resources and phase 4 has to do mainly with the establishment of contracts between enterprises.

Manifestation regards basically the individual profile definition and the manifestation of interest in participating in a VE by disseminating the profile. Creation/evolution involves in the first place the identification of a business opportunity. After this, a viability study that includes a rough plan for the VE should be made. Partner search and selection as well as the VE definition are the remaining steps. Configuration is the step were the VE processes are detailed and defined. VE processes have to do basically with inter-enterprise co-operative activities, which include the communicative elements definition such as formats and ontologies, as well as access rules. $\mathrm{VE}$ processes are configured in the creation phase of the VE, but reconfiguration is likely to be needed at some points of the VE life. 


\subsection{Operation}

In N/VE operation we are concerned with the execution of the distributed business processes. The main issue in this phase is to guarantee that the appropriate infrastructure (or execution system) is deployed to fully satisfy the design and configuration resulted from the creation phase.

Nevertheless, during the operation of a N/VE it might be necessary to do some adjustments (due to either operational or tactical reasons) such as change partners, include a new partner, change a partner role, etc. This originates a reconfiguration of the N/VE. This is a (re)design activity similar to the one in the creation phase.

\subsection{SAN approach to configuration/reconfiguration}

SAN role in the N/VE creation phase is mainly to give support to the network formation. This covers mostly the configuration step but also touches manifestation and evolution. Furthermore, some support can be given to reconfiguration activities in the operation phase. In the research work done so far we have found particularly useful the structural and relational levels of analysis.

\subsubsection{Structural analysis}

This analysis involves three steps: identification of the social actors, definition of the type(s) of SAN to be analysed and the modelling of the SAN.

The identification of relevant social actors cannot of course be dissociated from the type of analysis to be carried out. Furthermore, during the modelling and analysis, additional actors can be identified. This can be a complex topic and it is outside the scope of this paper. For now, it is satisfactory to consider as social actors the individuals or groups that are relevant in operational terms, i.e., the personnel, departments, etc., involved in the execution of the distributed business processes.

There are many types of analysis that can be done to support the configuration/reconfiguration of a N/VE. Again, the distributed business processes preliminarily defined set a first set of possible SAN. For example, if the N/VE is particularised in a supply chain, a logistic analysis (in a broad sense) is an obvious type of SAN to be considered (see figure 1). Less obvious would be a SAN depicting a "co-operative planning" process, involving several actors co-operating in responding to customer demands in the supply. 


\subsubsection{Relational analysis}

SAN Relational analysis focus on the relationship between two social actors in a SAN. The main goal is, within a given SAN, to characterise how two particular social actors interact. By classifying the links between nodes (either qualitatively or quantitatively) in any of the types above referred, it is possible to evaluate relationships against some pre-defined criteria. Examples of the later are "degree-of-interaction", "degree-of-control", "degree-of-dependency", "connection potential", "distance", etc.

In the example of figure 1, the relationship between the logistic dep. of Ent-A and logistic dep. of Ent-C is quite obvious in terms of co-operative planning for the supply chain. In the design of a co-operative planning business process for the N/VE several relational issues between the two social actors should be analysed for an optimised configuration of these processes. For example, operational issues (stated in the desired activity models for the business process) imply the exchange of production plans by the two departments. Consequently, either side should be able to access to this information timely and easily. Thus, in terms of configuring the N/VE, either of these actors should be characterised with an high level of accessibility regarding plans information.

On the other side, "co-operative planning" requires an attitude of trust between social actors (regarding particularly to information sharing). If the links in a network model this attitude, e.g. by assigning to each link a "degree-of-trust", this SAN could give the relative degree of trust between any two (connected) actors.

\section{EXAMPLE: SUPPLY CHAIN CO-OPERATION FOR ORDER ACCEPTANCE AND CHANGE}

\subsection{The CO-OPERATE project}

Co-OPERATE is an IST project supported by the European Commission, that aims at developing solutions to enhance the entire supply network, providing an advanced information and communication infrastructure to support general co-operation, as well as particular methodologies for co-operative planning and for network set-up and support. The project has the general goal of improving the performance of networked enterprises by achieving: co-operation and supply chain integration, higher responsiveness, lead time, reduction throughout the supply chain and reduced inventory levels, improved flexibility in the 
supply chain, increased capability to solve unexpected problems in the supply chain. In order to achieve these goals a number of "business solutions" (or general functionalities) have been defined, that will focus on the network aspects of the business processes and will try to accommodate and support the current internal tools and processes of companies. These business solutions are the following: feasibility studies for new order or change requests across the network, long term business planning for the network, standard operational order and planning processes, exception handling process, multi sourcing co-ordination, process visibility, performance management information.

\subsection{General requirements for a business solution}

In this work we are mainly concerned with the first of the above business solutions - ReFS - "feasibility studies for new order or change requests across the network". It should provide a set of functionalities that support the request for new orders or large order changes across the network and coordinate the feasibility checks at the individual companies within the shortest possible time. This includes checking of capacity and materials from suppliers. For the general functionality of ReFS, it will be considered a scenario that assumes that a core company with their own customers downstream in the chain, is customer of a number of suppliers. This company holds the order data and ensures the availability of them to their suppliers. The suppliers can request actualisation of orders either by a pull or push process. Information about orders should be sent automatically to all suppliers using the best communication channel. All orders from the core company to each supplier should be presented together with their actual status and the supplier should have the choice to view them sorted according to a customisable filter. All new and changed orders (which not yet have been acknowledged by the supplier) should be highlighted. The planner in the supplier companies should be able to acknowledge each new or changed order and register the acknowledgement data such as date/time, user identification, etc. All changes are stored in a history table. Feasibility checks at the individual companies should be made within the shortest possible time including checking for own capacities and requesting needed materials from suppliers, which need to do the same process themselves. This deals with the feasibility of acceptance of new orders, with the production of a quotation and with a real-time check of capacity and materials availability. Results should be co-ordinated and consolidated to come to a complete solution. This process achieves a co-operative production plan for the network. The end customer or a supplier, should be 
able to track, along the production chain, the positioning and the status of completion of each related order.

Figure 1 - A "logistic SAN" including IS agents

\subsection{SAN analysis for the business process configuration}

The first network developed to analyse this business solution was a "logistic" SAN (see figure 1). The structural analysis enabled, in the first place, a common understanding of how the actors involved would interact with each other in what concerns to the operational aspects of order acceptance and change.

\subsubsection{Information system specification}

We consider now the specification of the information system supporting

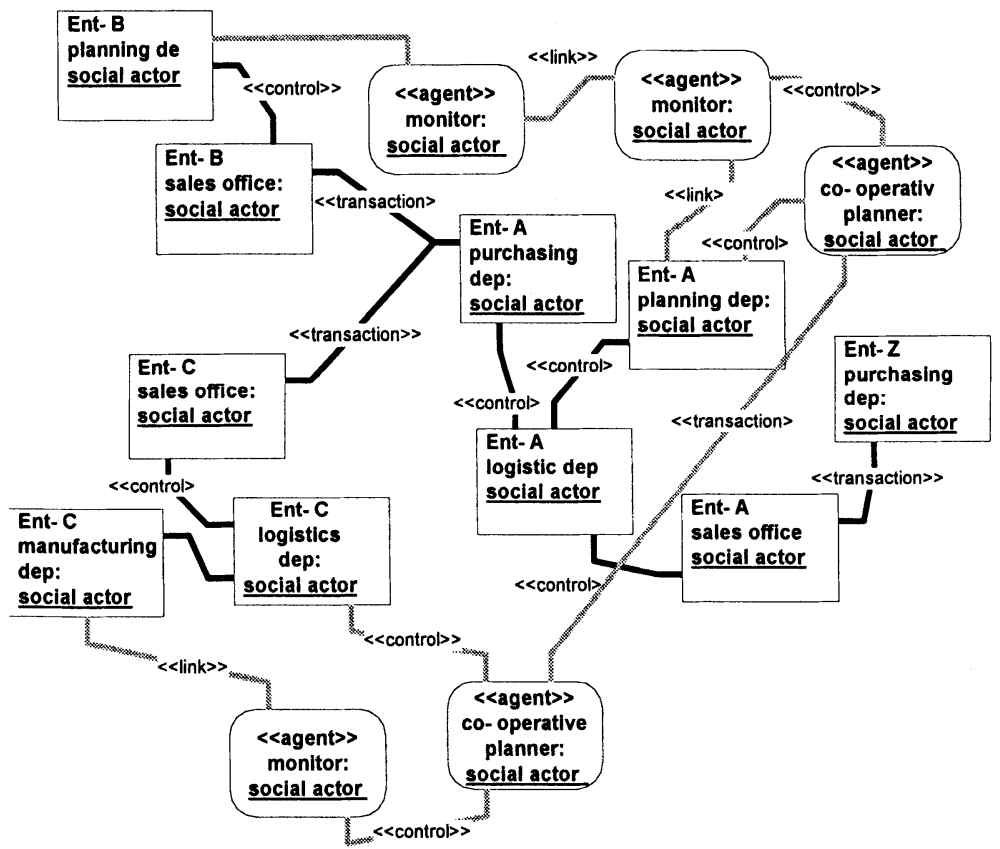

the "logistic" SAN of figure 5. A first specification of a community of agents would imply to define how the agents are conceptually integrated in the network. Two options can be considered:

1. Agents are social actors, i.e., are considered as behaving minimally as the human counterparts. This implies that agents establish relationships with human social actors and between themselves. 
2. Agents are extensions of the human social actors, modifying the characteristics and behaviour of the later. This leads to the modification of the types of relationships between social actors.

In a first approach we decided by the first option because it is easier to carry out the conceptual integration. Nevertheless, further research is needed in order to support with rigour this decision.

\subsubsection{Defining the infrastructure}

To support ReFS business process, an information system relying on a multi-agent infrastructure was specified. We considered this particular agent based system structured around the following basic architectural principles:

- each business unit in the network of companies is served by a set of agents;

- each of these sets forms a "node" in a community of agents distributed by the several "units" in the network;

- in each node, the agents co-operate to achieve local goals;

- in each node, each agent performs one or more functions, and coordinates its decisions with the other agents in the node;

- different nodes in the network co-operate to achieve global or local network goals; co-operation between nodes is carried out through the interactions of the individual agents in the different nodes;

- the functionality across the network or supply-chain is achieved through the interaction of the different nodes;

- the number and types of agents present in each node is variable, i.e. we can have all kind of agents or only some of them.

SAN models contemplating several aspects were built for this organisational reality (involving three companies in a supply-chain). The analysis of these models, together with technical UML based business models, led to a first specification of community of agents, identifying mainly the agent models and agent co-operation models.

\subsubsection{Basic types of agents}

From the above analysis, the required functionalities where assigned to a set of basic agent types (the new social actors). Basically, the design of the system was viewed as a set of roles that (1) interact with each other according to some protocol, (2) have responsibilities, which in a direct or indirect way determine the functionality inherent to that role, (3) have a set of permissions which identify the "rights" associated with the role, and 
subsequently identify the resources that are available to that role when its responsibilities are placed into practice, (4) perform local computations or activities (local in the sense that no interactions are carried out with any other role in the system).

Table 1 - Two basic agent types

\begin{tabular}{|c|c|}
\hline Agent & Role \\
\hline $\begin{array}{l}\text { Co- } \\
\text { operative } \\
\text { Planner }\end{array}$ & $\begin{array}{l}\text { - Co-operates with other planners in other nodes, in orde } \\
\text { to develop plans that achieve the network goals } \\
\text { - Interacts with the local Capacity Agent; }\end{array}$ \\
\hline Monitor & $\begin{array}{l}\text { - Acquires and aggregates the network or supply-chain } \\
\text { state variables; compares current state variables wit } \\
\text { target values; } \\
\text { - Identifies deviations or disruptions, e.g. resource failur } \\
\text { (loss of production capacity), logistics problems } \\
\text { (transport delays), quality issues; } \\
\text { - Propagates warnings to other agent types. }\end{array}$ \\
\hline
\end{tabular}

The resulting SAN is depicted in figure 1. This SAN shows clearly (in qualitative terms) the new relationships between the human social actors. For example, it can be concluded that Ent-A planning dep. will be able to better know what is happening in the suppliers, although potentially Ent-B would be "more accessible". Ent-C doesn't want (or can't) to make available information about the production plans and status.

\section{CONCLUSION AND FURTHER WORK}

This is a preliminary report of an on-going work. Although the conceptual tools provided by the approach revealed useful in complementing a distributed business process analysis and supporting information systems specification, there are more interesting possibilities open by a further sophistication of the network models. In particular, the use of graph theory can provide a set of quantitative results that can support the analysis even knowing that it is basically qualitative. Measures such as the "connection capital", "centrality", "groupability", can be calculated from the graph, if appropriate values are assigned to the connections. These measures will help to characterise in more detail the social actors and their relationships.

The example focused exclusively in the structural analysis. Current research work will result in a future paper describing how to fine tune the 
relationship between two social actors, using a relational analysis. In the same way, social interaction analysis is object of current research.

\section{References}

[1] Camarinha-Matos, L., Afsarmanesh, H., Rabelo, R., 2000, Supporting Agility in Virtual Enterprises, E-Business and Virtual Enterprises, Camarinha-Matos et al. (eds). Kluwer Academic Publishers.

[2] Lazega, E., 1997, Network Analysis and Qualitative Research: a Method of Contextualization, in G. Miller and R. Dingwall (eds.), Context and Method in Qualitative Research, London, Sage.

[3]Lemieux, V., 1999, Les réseaux d'acteurs sociaux, Presses Universitaires de France.

\section{Biographies}

António Lucas Soares is a senior researcher at INESC Porto and Assistant Professor at the Faculty of Engineering of University of Porto. César Augusto Toscano is project leader at INESC Porto.

Jorge Pinho de Sousa is a senior researcher at INESC Porto and Assistant Professor at the Faculty of Engineering of University of Porto. 